(C) by R. Oldenbourg Verlag, München 1997

\title{
Crystal structure of 1-benzyl-1,2,3-triazole, $\mathrm{C}_{9} \mathrm{H}_{9} \mathrm{~N}_{3}$
}

\author{
M. A. Sridhar, N. K. Lokanath, J. Shashidhara Prasad
}

University of Mysore, Department of Studies in Physics, Manasagangothri, Mysore 570 006, India

\author{
D. G. Bhadre Gowda and K. S. Rangappa
}

University of Mysore, Department of Studies in Chemistry. Manasagangothri, Mysore 570 006, India

Received May 28, 1996, CSD-No. 402515

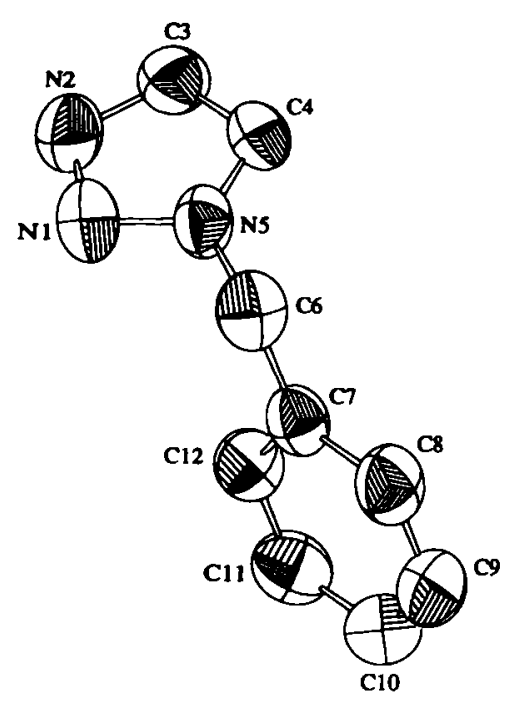

Fig. 1. Projection of the molecule on the best plane.

Source of material: synthesized (see ref. 1).

The bond distances and angles do not show any large deviations. The ORTEP plot of the molecule is shown in Fig. 1. The packing of the molecules down $b$ shown in Fig. 2.

C9H9N3, monoclinic, $P 1211$ (No. 4), $a=5.545(6) \AA$, $b=7.777(2) \AA, c=9.850(4) \AA, \beta=96.36(5)^{\circ}, V=422.2 \AA^{3}, Z=2$, $R(F)=0.043, R_{\mathrm{w}}\left(F^{2}\right)=0.130$.

Acknowledgment. The authors would like to express their thanks to DST, Government of India for financial assistance under the project SP/12/FOO/93.

Table 2. Final atomic coordinates and displacement parameters (in $\AA^{2}$ )

\begin{tabular}{|c|c|c|c|c|c|c|c|c|c|c|}
\hline Atom & Site & $x$ & $y$ & $z$ & $U_{11}$ & $U_{22}$ & $U_{33}$ & $U_{12}$ & $U_{13}$ & $U_{23}$ \\
\hline $\mathbf{N}(1)$ & $2 a$ & $0.4984(5)$ & $0.9531(5)$ & $0.6213(3)$ & $0.052(1)$ & $0.090(2)$ & $0.091(2)$ & $0.002(2)$ & $0.015(1)$ & $0.001(2)$ \\
\hline $\mathbf{N}(2)$ & $2 a$ & $0.5675(5)$ & $1.1113(5)$ & $0.6537(3)$ & $0.052(1)$ & $0.086(2)$ & $0.096(2)$ & $-0.007(2)$ & $0.008(1)$ & $0.013(2)$ \\
\hline$C(3)$ & $2 a$ & $0.3761(5)$ & $1.1974(5)$ & $0.6932(4)$ & $0.061(2)$ & $0.066(2)$ & $0.091(2)$ & $-0.001(2)$ & $-0.000(1)$ & $0.013(2)$ \\
\hline$C(4)$ & $2 a$ & $0.1814(5)$ & $1.0904(5)$ & $0.6850(3)$ & $0.048(2)$ & $0.066(2)$ & $0.078(2)$ & $0.009(1)$ & $0.003(1)$ & $0.007(2)$ \\
\hline$N(5)$ & $2 a$ & $0.2627(4)$ & $0.9409(4)$ & $0.6398(2)$ & $0.045(1)$ & $0.074(2)$ & $0.062(1)$ & $-0.001(1)$ & $0.0053(9)$ & $0.003(1)$ \\
\hline$C(6)$ & $2 a$ & $0.1339(6)$ & $0.7792(5)$ & $0.6150(3)$ & $0.064(2)$ & $0.076(2)$ & $0.068(2)$ & $-0.006(2)$ & $0.002(1)$ & $-0.012(2)$ \\
\hline$C(8)$ & $2 a$ & $-0.0631(6)$ & $0.5598(5)$ & $0.7522(4)$ & $0.056(2)$ & $0.077(2)$ & $0.094(2)$ & $-0.009(2)$ & $0.006(2)$ & $-0.008(2)$ \\
\hline$C(9)$ & $2 a$ & $-0.0682(6)$ & $0.4545(6)$ & $0.8635(4)$ & $0.066(2)$ & $0.075(2)$ & $0.114(3)$ & $-0.009(2)$ & $0.020(2)$ & $0.007(2)$ \\
\hline$C(10)$ & $2 a$ & $0.1206(6)$ & $0.4584(6)$ & $0.9675(4)$ & $0.084(2)$ & $0.071(2)$ & $0.092(2)$ & $0.004(2)$ & $0.015(2)$ & $0.012(2)$ \\
\hline$C(11)$ & $2 a$ & $0.3133(6)$ & $0.5666(5)$ & $0.9579(4)$ & $0.079(2)$ & $0.071(2)$ & $0.076(2)$ & $0.006(2)$ & $-0.004(2)$ & $-0.002(2)$ \\
\hline$C(12)$ & $2 a$ & $0.3201(5)$ & $0.6711(4)$ & $0.8455(3)$ & $0.060(2)$ & $0.062(2)$ & $0.075(2)$ & $-0.006(2)$ & $0.001(1)$ & $-0.007(2)$ \\
\hline
\end{tabular}

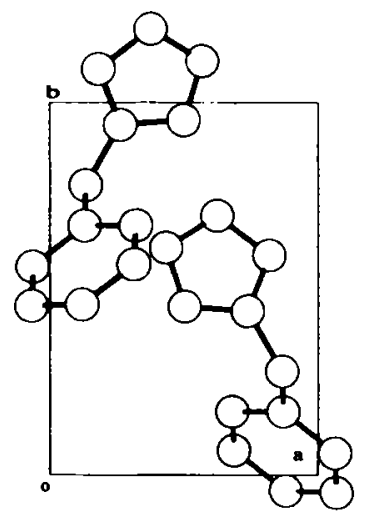

Fig. 2. Packing of the molecules down $b$.

Table 1. Parameters used for the X-ray data collection

$\begin{array}{ll}\text { Crystal: } & \text { white, rectangular, size } 0.20 \times 0.25 \times 0.35 \mathrm{~mm} \\ \text { Wavelength: } & \text { Mo } K_{\alpha} \text { radiation }(0.71069 \AA) \\ \mu: & 0.80 \mathrm{~cm}^{-1} \\ \text { Diffractometer: } & \text { Rigaku AFC7S } \\ \text { Scan mode: } & \omega / 2 \theta \\ T_{\text {measurement: }} & 293 \mathrm{~K} \\ 2 \theta_{\text {max: }} & 50^{\circ} \\ \text { N(hkl) } & 805 \\ \text { Criterion for } I_{0}: & I_{0}>2 \sigma\left(I_{0}\right) \\ \text { N(param) } & 110 \\ \text { Programs: } & \text { SHELXS-86, SHELXL-93 }\end{array}$

\section{References}

1. Bhadre Gowda, D. G.: Synthesis and crystal structure of some nitrenium compounds. Ph. D. Thesis, University of Mysore, India 1997.

2. Sheldrick, G. M.: Phase annealing in SHELX-90: Direct methods for larger structures. Acta Crystallogr. A46 (1990) 467-473.

3. Sheldrick, G. M.: SHELXL-93. Crystal structure refinement program. University of Göttingen, Germany. 